\title{
TRAJECTORY-BASED COMPLEXITY (TBX): A MODIFIED AIRCRAFT COUNT TO PREDICT SECTOR COMPLEXITY DURING TRAJECTORY-BASED OPERATIONS
}

\author{
Thomas Prevot, NASA Ames Research Center, Moffett Field, CA \\ Paul U. Lee, San Jose State University/NASA Ames Research Center, Moffett Field, CA
}

\begin{abstract}
In this paper we introduce a new complexity metric to predict -in real-time- sector complexity for trajectory-based operations (TBO). TBO will be implemented in the Next Generation Air Transportation System (NextGen). Trajectory-Based Complexity (TBX) is a modified aircraft count that can easily be computed and communicated in a TBO environment based upon predictions of aircraft and weather trajectories. TBX is scaled to aircraft count and represents an alternate and additional means to manage air traffic demand and capacity with more consideration of dynamic factors such as weather, aircraft equipage or predicted separation violations, as well as static factors such as sector size. We have developed and evaluated TBX in the Airspace Operations Laboratory (AOL) at the NASA Ames Research Center during human-in-the-loop studies of trajectory-based concepts since 2009. In this paper we will describe the TBX computation in detail and present the underlying algorithm. Next, we will describe the specific TBX used in an experiment at NASA's AOL. We will evaluate the performance of this metric using data collected during a controller-inthe-loop study on trajectory-based operations at different equipage levels. In this study controllers were prompted at regular intervals to rate their current workload on a numeric scale. When comparing this real-time workload rating to the TBX values predicted for these time periods we demonstrate that TBX is a better predictor of workload than aircraft count. Furthermore we demonstrate that TBX is well suited to be used for complexity management in TBO and can easily be adjusted to future operational concepts.
\end{abstract}

\section{Introduction}

The Next Generation Air Transportation System (NextGen) is being developed to transform the current system to the one envisioned for the future by increasing capacity/throughput, improving flight and system efficiency, all while maintaining overall system safety [1]. A number of human-in-the-loop studies have been conducted in the Airspace Operations Laboratory (AOL) at the NASA Ames Research Center to evaluate mid-term NextGen concepts [2, 3]. NextGen concepts such as MultiSector Planning and Dynamic Airspace Configuration required the use of a sector complexity estimate instead of using a simple aircraft count. Although predicted aircraft count is used in today's air traffic management to manage a maximum traffic threshold, it is well known that aircraft count alone is not the best predictor of traffic complexity [4]. In addition, mid-term NextGen environments are expected to have diverse avionics and ground equipage, which further complicate the relationship between simple aircraft count and controller workload, making the need for new complexity metrics vital.

Although various complexity metrics have been proposed and studied over the past decades [e.g., 411], they were unsuitable for use during our real-time human in the loop simulations. One of the main reasons was that many complexity computations were computed off-line and matched to previously collected data rather than in real-time based upon current and predicted data. The resulting metrics that provided good statistical fit to the historical data were often ill-suited to characterize the uncertainties associated with traffic predictions. Another shortcoming of many complexity metrics was that they were not easily understood by human operators in ways that allowed them to interpret the data and translate them into actions that support air traffic management. Finally, the existing metrics were often designed for current-day operations rather than trajectory-based operations (TBO) planned for NextGen.

A new Trajectory-Based Complexity (TBX) was developed to support NextGen concept evaluations in NASA's AOL. TBX has many similarities to past 
approaches in the complexity research but its implementation is different in many ways. In the following sections, past approaches to calculating traffic complexity are described, followed by the details of TBX calculations.

\section{Background}

In today's air traffic operations, a sector's predicted aircraft count is used to determine the maximum traffic threshold that a controller can handle in that sector. The maximum threshold value is called Monitor Alert Parameter (MAP) and its value (i.e. maximum aircraft count in a sector) is set based on average sector flight time. However, the MAP values and the predicted aircraft count alone have been insufficient to correctly measure controller task loads without considering other factors that vary the traffic complexity [5].

One of the main efforts in traffic complexity research is targeted towards developing Dynamic Density (DD) metrics. It is a method to identify numerous factors that contribute to traffic complexity and that correlate with controller workload. A large set of metrics have been proposed as DD metrics [4]. Although DD metrics correlated better with controllers' workload ratings than the aircraft count alone, the total number of DD metrics initially exceeded fifty and the number still remained above ten after further down-selection. Simplified Dynamic Density (SDD) was another way to down-select a subset of DD metrics to those that were readily available in the current Enhanced Traffic Management System (ETMS) [6]. The identified SDD metrics were sector occupancy counts, proximities in a sector, altitude transitions in a sector, transfers across sector boundaries, number of aircraft per sector volume, variance of aircraft headings in sector, and variance of cruising aircraft speeds in sector.

Despite the better prediction of traffic complexity and controller workload, regression methods that fit the controller workload to different DD metrics are only valid for the fitted sectors. The weightings of the factors change significantly for different sectors and the methodology offers no easy solution to modify the factor weightings to adapt the past calculations to new sectors. Furthermore, many of the DD factors are not suited for traffic flow management (TFM) [7]. If DD metrics exceed the maximum threshold, it is not obvious which actions should be taken in order to solve the problem [7]. Some of the DD metrics also exhibit large uncertainty when they are projected to the TFM time horizon (e.g. one to two hours), which results in significant fluctuations of the combined complexity values [8].

A different approach to measuring traffic complexity is to select a few complexity factors that the air traffic controllers can readily identify and solve within the TFM time horizon. For example, Masalonis and his colleagues [7] asked five Traffic Management Coordinators (TMCs) various workload factors that they considered in their decision making. The factors that they identified included predicted peak aircraft count, percentage of sector capacity lost due to severe weather, weather at the destination airport, traffic at same altitude on merging/crossing flows, impact of weather on other surrounding sectors, total aircraft count, arrival and departure push near a sector, traffic at same altitude with lateral proximity, and amount of time the sector stays above MAP.

In a similar approach, Hilburn and Flynn identified complexity factors for the Complexity and Capacity (COCA) project [9]. They identified the following factors based on the discussions with controllers: a mix of climbing and descending aircraft, turbulence/weather, military/other restricted areas, traffic flows converging at the same point, traffic bunching, radio congestion, high number of aircraft, multiple crossing points, mix of climbing and descending flights in cruise, crossing points close to boundaries, merging of arrival flows, and mix of high and low performance aircraft. They also identified some of these factors as "precursor" factors, whose presence elevates the complexity of other factors. Some of the pre-cursor factors identified by the controllers were frequency congestion, mix of climb/descent traffic, high number of aircraft, emergencies, military/other restricted areas, turbulence/weather, and non-nominal equipment status.

Histon and Hansman [10] proposed three categories of ATC complexity: situation, perceived, and cognitive complexity. They define situation complexity as an intrinsic property of the traffic situation; perceived complexity as subjective complexity experienced by the controllers; and 
cognitive complexity as the complexity of the mental models and strategies that controllers use to perform air traffic management tasks. They then focus their research on cognitive complexity which drives controller workload [10].

In a series of follow-up studies, Hansman and his colleagues proposed an integrated metric called Modified Aircraft Count (MAC), which consists of combinations of factors that account for different components of cognitive complexity [11]. The MAC metric is calibrated to represent the effective number of aircraft in a sector as a replacement for today's MAP which is based on the predicted aircraft count. The MAC is a combination of each aircraft's contribution to cognitive complexity (called Aircraft Multiplier, or AM), which is then multiplied by sector level complexity (called Sector Multiplier, or SM), such as its shape, Letters of Agreements (LOAs), etc. Examples of AM complexity factors are aircraft proximity, sector boundary encounters, weather impacting area encounters, climbing/descending aircraft, location relative to critical points, and communication capability (e.g. Data Comm or voice). Examples of $S M$ factors are airspace volume, number and position of ingress/egress points, spatial distribution of airways, and traffic restriction (e.g. metering).

\section{TBX overview}

In our complexity calculations, we tried to avoid some of the pitfalls of prior approaches while keeping some of the features that would work well for predicted complexity calculations that human operators could use for TFM purposes. The characteristics of these features were:

- Metrics that were comprehensible to human operators

- Metrics whose weightings would be easy to modify for new equipage and operational environments

- Metrics that were stable over the prediction time horizon

- Use of TBO to create more stable trajectory predictions over a $1-2$ hour time horizon

- Combination of different complexity factors into a single modified aircraft count value that human operators could use in conjunction with a MAP value

In order to be able to support real-time TFM, TBX is designed for real-time computations of complexity estimates based upon predictions of fourdimensional trajectories for weather and aircraft. TBX accounts for the differences in controller task load associated with maintaining TBO for different equipage levels, and the increased complexity associated with climbing and descending aircraft. The factors that provide inputs to TBX calculations were obtained from discussions with subject matter experts and therefore were easily accessible and understandable by the operators. As a real-time estimate, TBX can provide immediate what-if feedback on the complexity impact of potential trajectory and/or airspace changes. This allows traffic managers and area supervisors to preview the impact of potential solutions to capacity/demand imbalances before deciding on and implementing a specific strategy.

Calculating the TBX value is based upon establishing capacity definitions for nominal conditions, comparing the currently predicted conditions to those nominal conditions, and then adjusting the aircraft count accordingly. Thus, TBX values mimic aircraft count and the same mechanism of setting capacity thresholds using a MAP that reflects the maximum aircraft count can be used with TBX. The TBX value will be equal to the predicted aircraft count for a "nominal" sector size, a nominal number of transitioning aircraft and a nominal equipage mix, but any predicted difference to nominal operations, such as convective weather cells, different aircraft equipage mixes, unusually high levels of transitioning aircraft or traffic conflicts, causes a modification to the aircraft count, which will be reflected in the respective TBX value. Using Figure 1we will illustrate the use of TBX.

Figure 1 shows excerpts from a traffic situation display (top), a load table (bottom left) and a load graph (bottom right) to illustrate an example from a simulation run in the Kansas City Center (ZKC) airspace. The traffic situation display indicates three sectors, marked 94, 98 and 90, with a weather system present in sector 94. The load table shows the predicted peak aircraft count and TBX value of these three sectors for the next three 15 minute time periods. The peak aircraft count is the upper number 
in each cell. TBX, the predicted complexity, is the lower number in each cell. The load graph shows the predicted TBX value for sector 94 for the next 75 minutes. In this simulation, which assumed a midterm environment with many data link equipped aircraft and several decision support tools for the controllers, the MAP for each sector was set to 24 instead of today's 18 .

Based upon aircraft count alone, ZKC_98 and ZKC 94 would not reach nor exceed the MAP value. TBX however predicts that in 15 to 30 minutes both sectors will show a similar workload for the controllers as 25 or 28 aircraft would under normal conditions. The reason is that the weather system impacts aircraft in both sectors and requires reroutes. Furthermore, sector ZKC 98 is a small sector with

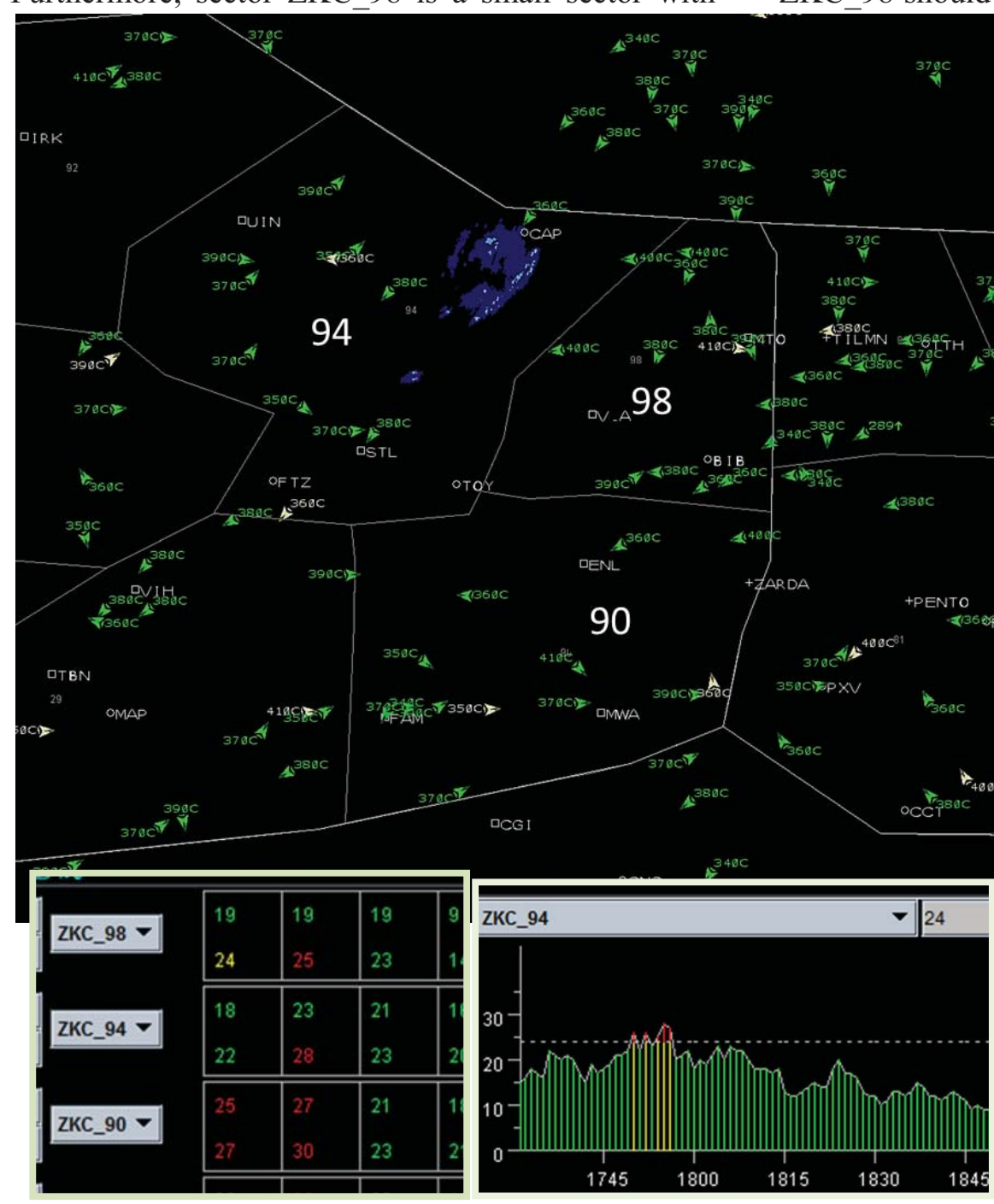

Figure 1: Traffic situation, load table and load graph many transitioning aircraft that makes it generally more complex than ZKC_94. Because more aircraft are diverted around the weather, ZKC_90, which currently does not look very complex, will be subject to dense traffic flows entering form the north east that create additional conflicts and will make the high number of aircraft predicted to enter even less manageable.

In this situation, a traffic management coordinator or area supervisor looking at aircraft count alone would be mostly concerned with sector ZKC_90 and perhaps reroute aircraft into ZKC_98. However, using TBX it becomes clear that all three sectors will be saturated (i.e., red numbers in load table); and to compensate for this, ZKC 94 and ZKC 98 should be managed with R-Side and D-Side controller teams. Two to three more aircraft might need to be moved, so they will not enter into ZKC_90, ZKC_98 and ZKC_94. This will bring the TBX values for each sector down to nominal levels.

As the example indicates, TBX is based upon comparing predicted conditions to nominal conditions and making adjustments to the actual aircraft count that reflect the differences. The result can be interpreted as a "feels like" value. In the example in Figure 1, there are 18 aircraft predicted to be in sector ZKC_94, but it may feel to the controller like 22 aircraft, because the workload to manage 18 aircraft in a weather impacted sector may be similar to the workload associated with managing 22 aircraft under nominal conditions. The TBX computation takes weather penetration into account and as the TBX value in ZKC_94 reaches 28, it exceeds the monitor alert parameter of 24 set for this sector. Therefore, the TBX value of 28 indicates that the sector will be overloaded. The load graph at the bottom right of Figure 1 indicates 
exactly how long this situation will prevail, thereby giving the operator more information. This information may help them to decide whether the sector team can handle the situation or whether aircraft will need to be rerouted.

In the preceding section we tried to demonstrate the value in using TBX in addition to aircraft count. The following sections will illustrate the TBX calculation approach step by step.

\section{TBX calculation}

The TBX calculation consists of two a-priori determinations prior to being computed in real-time:

1. Define nominal conditions, in which aircraft count is a good predictor of controller workload

2. Define adjustments to the nominal conditions that capture the differences in complexity between the current conditions and the nominal conditions.

3. Compute the TBX value

\section{Nominal Conditions}

The nominal conditions are those conditions under which the aircraft count is an excellent predictor of sector complexity. These are the nominal conditions within a sector and can be defined through knowledge elicitation sessions on a sector by sector basis or based upon more generic attributes. Up until now we used TBX for the high altitude airspace and we defined nominal conditions for that environment with only small variations for each simulation. For our simulations, we have defined a nominal sector to be a square $100 \mathrm{nmi}$ by $100 \mathrm{nmi}$ sector with a nominal number of aircraft in the sector to be $80 \%$ of the MAP value. It is also defined to have $10 \%$ of all aircraft with predicted loss of separation within the next 8 minutes and $20 \%$ of the aircraft to be climbing or descending. In the nominal conditions no aircraft are expected to penetrate weather. Nominal equipage mixes and MAP values depend on the simulated NextGen environment. For current day operations our nominal equipage mix has $0 \%$ Data Comm equipped aircraft and a MAP value of 18 . For a mid-term environment, we may assume $40 \%$ equipped aircraft and a MAP value of 23 and for a far-term more advanced environment we may assume 100\% equipage and a MAP value of 45. TBX allows subject matter experts to define the nominal conditions easily that will help relate aircraft count, TBX and MAP.

\section{Adjustments}

Adjustments are ratios that describe the relationship between a nominal value and the current value. In line with prior research and SME interviews there are two types of adjustments that should be made to the aircraft count to account for the different properties of the complexity impacts:

1. Primary (or dominant) complexity adjustments - those factors that can cause a similar effect to the complexity as the aircraft count, can offset the aircraft count, or have a detrimental impact. These factors are expected to have a multiplicative effect on the aircraft count and their impact is expected to scale with the current aircraft count. Primary adjustments are made for dominant items such as weather or sector size.

2. Secondary complexity adjustments - those factors have a secondary impact on complexity. They can be specific to a given operational environment and have a smaller impact on complexity than the primary factors. Their impact is less dependent on the overall aircraft count. Secondary adjustments are made for items such as number of conflicts, or number of transitioning aircraft.

\section{Computation}

In its general form we compute the TBX value as shown in equation (1). The TBX value of a sector $s$ at time $t$ equals the sum of its predicted aircraft count (ac) multiplied by the product of the primary adjustments (px) and the nominal aircraft count multiplied by the weighted sum of the secondary adjustments (sx) -1 .

TBX and its adjustments are defined such that their value under nominal conditions is 1 . This satisfies equation (2) below, which simply states that the TBX value of a sector $s$ at time $t$ under nominal conditions equals its predicted aircraft count at time $t$. In addition, all adjustments $\mathrm{px}_{\mathrm{i}}(\mathrm{s})$ and $\mathrm{sx}_{\mathrm{j}}(\mathrm{s})$ are limited to a range of 0 to 2 to prevent one factor from dominating the overall TBX value. 


$$
\begin{gathered}
t b x(s, t)=a c(s, t) * \prod_{i=0}^{n} p x_{i}(s, t)+a c_{n o m}(s) *\left(\frac{\sum_{j=0}^{m}\left(w_{j} * s x_{j}(s, t)\right)}{\sum_{j=0}^{m}\left(w_{j}\right)}-1\right) \\
t b x_{n o m}(s, t)=a c(s, t)
\end{gathered}
$$

where

$s \quad$ is the sector of interest

$t \quad$ is the time for which the complexity is computed

$\operatorname{tbx}(s, t)$ is the predicted trajectory-based complexity of sector $s$ at time $t$

$a c(s, t) \quad$ is the predicted aircraft count of sector $s$ at time $t$

$p x_{i}(s, t) \quad$ is the adjustment for primary complexity item $i$ in sector $s$ at time $t$

$a c_{\text {nom }}(s)$ is the nominal aircraft count in sector $\mathrm{s}$

$w_{j} \quad$ is the weight of secondary complexity item $\mathrm{j}$

$s x_{j}(s, t) \quad$ is the adjustment for secondary complexity item $\mathrm{j}$ in sector $s$ at time $t$

By setting up the calculations in this fashion, we can define the MAP values based on nominal conditions for a given sector and then compare aircraft count and TBX value relative to the MAP to assess the peak that exceeds the MAP. This method of presenting TBX values in the same format as aircraft count makes the interpretation of TBX easy for controllers.

\section{Complexity Factors}

After having presented the mathematical formulation of TBX, and before diving into specific examples and results, in this section we review the main complexity factors that we used. While many complexity factors exist, not all of them are useful, nor can or should they be used for all operational environments.

Complexity factors for TBX need to be meaningful to the operators and predictable for computers. In knowledge elicitation sessions with subject matter experts the following short list of complexity factors for a mixed equipage en route airspace was composed:

- Aircraft count

- Weather

- Sector volume

- Number of transitioning aircraft
- Conflicts

- Aircraft equipage

These factors have evolved over the years as the different simulation studies focused on some of the factors more than others. The actual calculations have been modified as well in response to how well they functioned in the studies. As we find better mathematical representations of the factors based on their efficacy in future studies, we expect to further modify and refine the complexity calculations. In the following sections, the calculations of the current complexity factors will be described more in detail.

\section{Aircraft Count}

TBX is based upon the assumption that aircraft count is the best predictor of workload under nominal conditions. Therefore, the aircraft count is a dominant factor and equation (1) makes this relationship explicit. Equation (2) on the previous page requires that the adjustment for the aircraft count needs to be linear. Therefore the aircraft count adjustment is simply

$$
p x_{a c}(s, t)=a c(s, t)
$$

Where

$p x_{a c}$ is a primary complexity adjustment for the aircraft count 
$s$ is the sector of interest

$t$ is the time for the prediction

$a c(s, t)$ is the predicted aircraft count at time $\mathrm{t}$

This relationship is already explicitly included in equation (1) and is stated here for completeness.

\section{Sector Volume}

The sector volume can be an important contributor to complexity since larger sectors provide more airspace than smaller sectors for maneuvering, more time between accepting and initiating handoffs and their associated transfers of communication. We currently use the following adjustment for sector volume:

$$
p x_{s v}(s, t)=\left(\frac{s v(s, t)}{s v_{\text {nom }}}\right)^{.15}
$$

where

$p x_{s v}$ is a primary complexity adjustment for the sector volume

$s v$ is the sector volume of the current sector (between its lateral and vertical boundaries)

$s v_{\text {nom }}$ is the nominal sector volume and

Our current nominal sector is

$$
s v_{\text {nom }}=100 \mathrm{nmi} * 100 \mathrm{nmi} * 10,000 \mathrm{ft}
$$

The power of 0.15 has been tuned empirically using prior studies by assessing the impact of sector size on the overall complexity value and comparing the complexity to the corresponding controller workload. As with all our adjustments this sector volume equation is a simple approximation that works reasonably well for sectors of comparable properties. Other options are to use different functions, to adapt the sector adjustment per sector or to scale the MAP value to reflect a specific sector more appropriately.

\section{Weather}

Weather impacts complexity in many ways. It limits the available airspace, causes the need for rerouting flights and often results in substantial increases in communication between pilots and controllers. Weather also becomes less predictable the further out into the future the prediction is made. For our TBX calculation we decided to use the number of aircraft that are predicted to penetrate the weather within a given sector. Therefore the predicted weather trajectory is compared against the predicted aircraft trajectory to determine whether these two intersect within a sector. We compute the complexity adjustment for weather as follows:

$$
p x_{w x}(s, t)=1+2 * \frac{a c_{w x}(s, t)}{M A P}
$$

where

$p x_{w x}$ is a primary complexity adjustment for the weather penetrations

$a c_{w x}$ is the number of aircraft predicted to penetrate the weather

\section{MAP is the Monitor Alert Parameter}

Weather is used as a primary adjustment and multiplies the aircraft count. Therefore the equation above means that if half of a sectors MAP value will penetrate the weather, the complexity is doubled in comparison.

\section{Number of Transitioning Aircraft}

The number of climbing and descending aircraft impacts the complexity in multiple ways. Controllers have to issue additional instructions; a larger amount of airspace needs to be clear of traffic while the aircraft are transitioning and the future locations of aircraft are more difficult to predict by humans and the trajectory automation because of large differences in aircraft performance. We integrate the number of transitioning aircraft as a secondary adjustment that scales independent of the actual aircraft count as follows.

$$
s x_{t r s}(s, t)=1+\frac{a c_{t r s}(s, t)}{M A P}-\operatorname{tr} s_{n o m}
$$

where

$s x_{t r s}$ is a secondary complexity adjustment for transitioning aircraft

$a c_{t r s}$ is the predicted number of aircraft to be transitioning

$\operatorname{trs} s_{n o m}$ is the nominal ratio of aircraft to be transitioning

We used $\operatorname{tr} s_{\text {nom }}=0.2$ for our high altitude environment, which means that in the nominal case $20 \%$ of the sectors MAP value (usually 4-5 aircraft) are transitioning 


\section{Conflicts}

Conflicts between aircraft add to complexity, because controllers have to assess the situation and maneuver at least one of the conflicting aircraft. This often also involves coordinating with adjacent sectors, further increasing the workload. Conflicts can be predicted by trajectory automation, but because of uncertainties, only reliably for up to 20 or 30 minutes. Therefore conflicts contribute to complexity calculations up to the conflict probes look ahead time (usually no more than 30 minutes) but they are excluded in the calculations beyond that.

We consider two types of conflict related data separately: The number of loss of separation (LOS) events that are predicted to occur inside the sector of interest and the number of aircraft that have a predicted conflict somewhere downstream (whether inside or outside of the sector of interest).

\section{Number of Predicted LOS events}

Conflicts that are predicted to occur within the sector are included as follows:

$$
s x_{\operatorname{PrLos}}(s, t)=1+2 *\left(\frac{\operatorname{PrLos}(s, t)}{M A P}-\operatorname{PrLos} \operatorname{Lnom}\right)
$$

where

$s x_{\text {PrLos }}$ is a secondary adjustment for the predicted number of LOS that occur in sector $\mathrm{s}$ at time $\mathrm{t}$

$\operatorname{PrLos}(s, t)$ is the number of predicted LOS events to occur in sector $s$ at time $t$, if no action is taken

$\operatorname{PrLos}_{n o m}$ is the nominal value for predicted LOS to occur in a sector at time $\mathrm{t}$

We used $\operatorname{PrLOS}$ nom $=0.05$ for our high altitude environment, which means that in the nominal case the number of conflicts is about as much as $5 \%$ of the sectors MAP value. For near-term MAP values of $\sim 20$, we expect one conflict to be predicted in the sector at any time

\section{Number of Aircraft with Predicted Conflicts}

The number of aircraft with predicted conflicts is different than the number of predicted LOS events as these LOS events can occur in a different sector. It is assumed that in a trajectory-based environment these would be flagged to the controller. We compute the complexity adjustment for this factor as follows:

$$
s x_{\text {conf }}(s, t)=1+\frac{a c_{\text {conf }}(s, t)}{M A P}-\operatorname{conf} f_{\text {nom }}
$$

where

$s x_{\text {conf }}(\mathrm{s}, \mathrm{t})$ is a secondary complexity adjustment for the number of aircraft with predicted conflicts in sector $\mathrm{s}$ at time $\mathrm{t}$

$a c_{\text {conf }}(\mathrm{s}, \mathrm{t})$ is the predicted number of aircraft in sector $\mathrm{s}$ at time $\mathrm{t}$ that are predicted to have a conflict at or after time $t$

conf $f_{\text {nom }}$ is the nominal ratio of aircraft with predicted conflicts per MAP

We used $\operatorname{con} f_{\text {nom }}=0.1$ for our high altitude environment, similar to the complexity adjustments for predicted LOS

\section{Aircraft Equipage}

Aircraft equipage is meaningful if there are equipage differences that lead to much different workload levels associated with the equipage types. In our studies, which were situated in a mid-term environment, we typically distinguished between aircraft that can be controlled via Data Comm. and those that have to be controlled via voice. Since we simulate automated transfer of communication for Data Comm equipped aircraft and trajectory uplinks they require significantly less work than unequipped aircraft. We adjust the complexity for equipage as follows:

$$
s x_{\text {uneq }}(s, t)=1+\frac{a c_{\text {uneq }}(s, t)}{a c(s, t)}-\text { uneq }_{\text {nom }}
$$

where

$s x_{\text {uneq }}(s, t)$ is a secondary complexity adjustment for unequipped aircraft in sector $\mathrm{s}$ at time $\mathrm{t}$

$a c_{\text {uneq }}(s, t)$ is the predicted number of unequipped aircraft in sector $\mathrm{s}$ at time $\mathrm{t}$

$a c(s, t)$ is the predicted number of aircraft in sector $\mathrm{s}$ at time $\mathrm{t}$

$u n e q_{n o m}$ is the nominal ratio of unequipped aircraft 
We use $u n e q_{n o m}=1$ (all aircraft unequipped) for our near-term environment, une $q_{\text {nom }}=0.5(50 \%$ equipped) for our mid-term mixed equipage environment, and $u n e q_{n o m}=0$ for our far-term full equipage environment. This value needs to be selected according to the target environment

The factors above represent the general factors that we use for computing the TBX. This exact set was used for mixed equipage simulations that investigated flow-based trajectory management and multi-sector planning [3, 13]. During these simulations, TMCs and area supervisors were highly successful in providing service for equipage and managing the workload in weather impacted sectors. Next we will discuss an example of a specific TBX formulation and results from our most recent study on Corridors-In-The-Sky.

\section{Example: Corridors-In-The-Sky}

\section{Study Background}

From July 11-22, 2011, a human-in-the-loop (HITL) simulation of the Corridors-In-The-Sky concept was conducted in the AOL This HITL study focused on investigating the feasibility and the potential benefits of this concept with air traffic controllers in a realistic environment.

As shown in Figure 2, the airspace in the study consisted of super high en route airspace sectors (FL330 and above) in the Cleveland Center airspace (ZOB). The corridors were designed as one-way routes westbound from or eastbound to the New York Metro area at two different altitudes. Each corridor had two parallel lanes to accommodate different speeds of aircraft.

The corridors were managed by the sector team within their respective sectors and were not treated as separate airspace. There was a mix of aircraft with and without Data Comm. Equipped aircraft had Data Comm technologies for uplinks of route, altitude, speed, and transfer of communication. Unequipped aircraft had no Data Comm and required voice instructions by the controllers. All aircraft were equipped with flight management systems with area navigation (RNAV) capability.

Feasibility and benefits were tested for four different corridor structures: (1) no specific corridors (No Corridors), (2) only equipped aircraft within

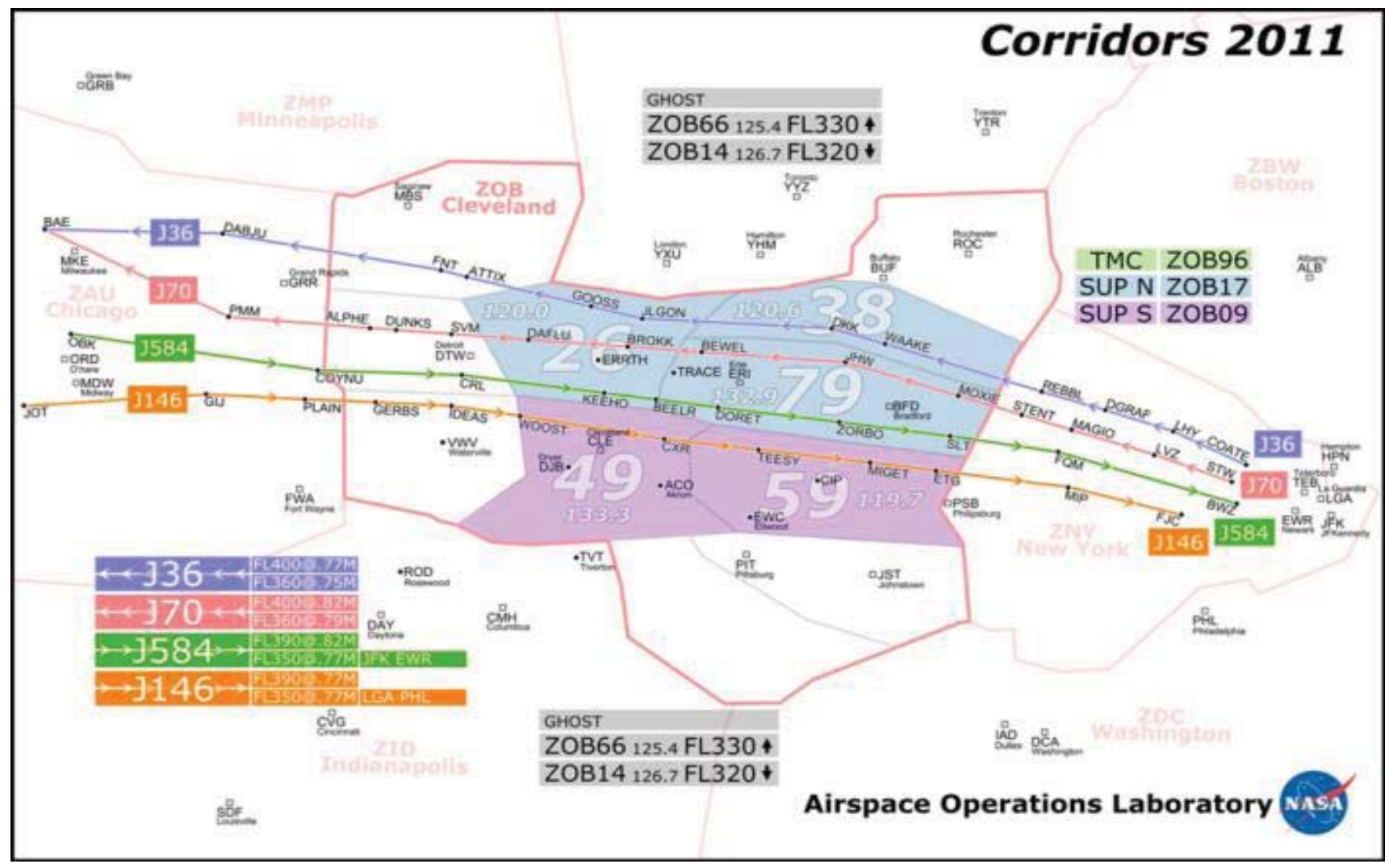

Figure 2: Corridors study test airspace 


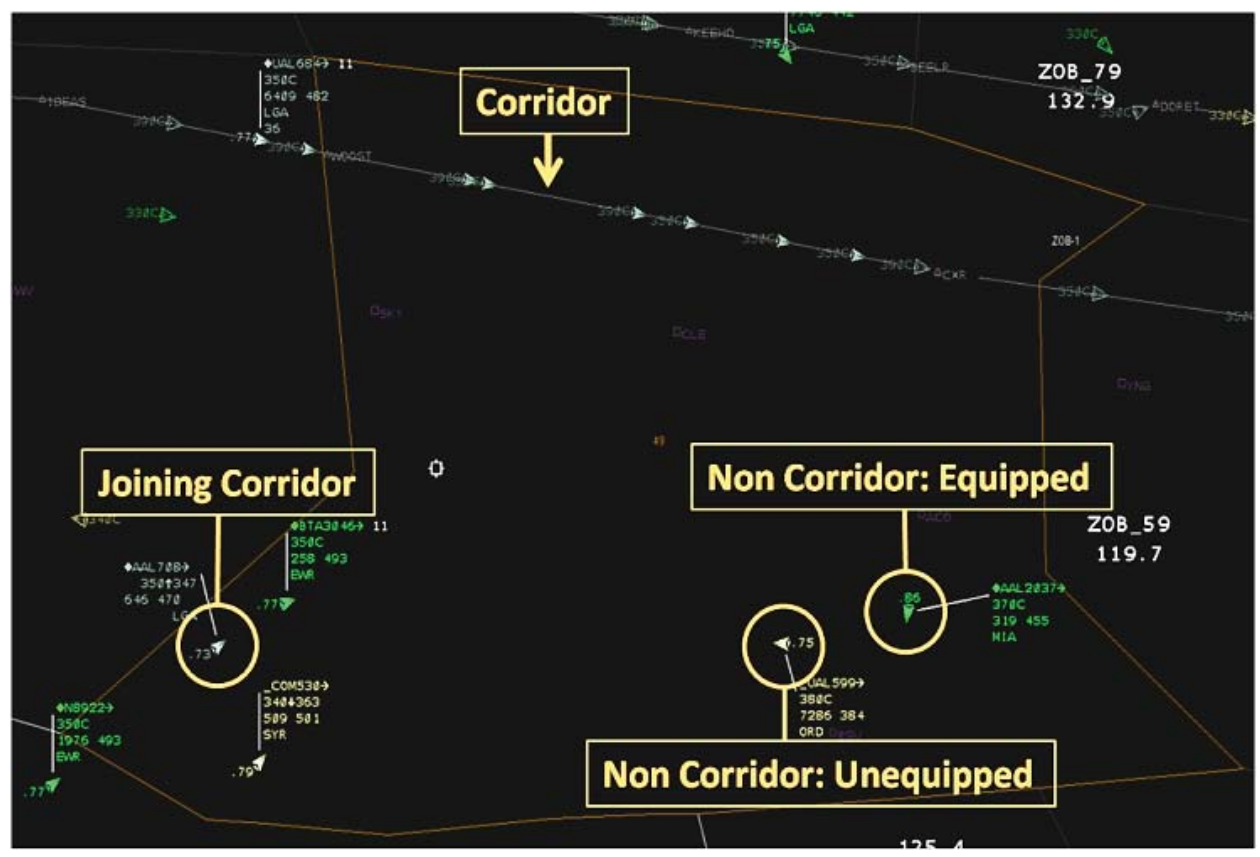

Figure 3: Controller display with active corridors and surrounding mixed equipage traffic

corridors (Equipped in Corridors), (3) only unequipped aircraft within corridors (Unequipped in Corridors), and (4) a mix of both equipped and unequipped aircraft within corridors (Mixed in Corridors). Surrounding non-corridor traffic consisted of 50/50 mix of Data Comm and non-Data Comm equipped aircraft in all conditions.

\section{TBX usage during study}

Prior to the study, a MAP value of 22 was established as the maximum traffic that controllers could handle under nominal condition. Therefore, TBX had to be defined to accommodate the expected workload differences in the various conditions and the different states and Data Comm equipage types of the aircraft compared to the nominal condition. The next two paragraphs explain the operational environment and the differences that had to be captured in the TBX computations.

In the study, the traffic managers and supervisors were told to use TBX to reroute aircraft. Traffic Managers were told to keep the TBX value at or below 25. Supervisors managed the remaining complexities towards a TBX value of 22 , which was understood to be an approximately match to the maximum traffic that controllers could handle.
Voice communications were available at all times between controllers and pilots. Conflict detection automation was active and resolution support was available. For Data Comm equipped aircraft, clearances were sent either via data communications and loaded into the FMS, or via voice in which the pilot manually performed the required actions. Handoffs and transfers of communication for Data Comm equipped aircraft were automated and did not require controller involvement. For unequipped aircraft, clearances were only sent via voice and were typically followed by controller actions to update the system accordingly. Handoff initiations were automated, but acceptance and transfer of communications were performed manually by the controller.

Non-corridor traffic within the test sectors was displayed with full data blocks and was collapsible only after being handed off and exiting the sector. Equipped aircraft were displayed in green while unequipped aircraft were displayed in yellow (see Figure 3). For aircraft in the corridors, data blocks regardless of equipage were collapsible at any time. By default, equipped aircraft in the corridors were displayed with collapsed data blocks. The data blocks of unequipped aircraft in the corridors popped up when in hand off status. The colors used for corridors 
traffic were muted variants of the green and yellow used to denote the equipage of non-corridors traffic. Additional information was also included in corridor traffic data blocks that indicated time to entry or exit of corridors, and the assigned corridor for entering traffic.

\section{Additional TBX adjustments for the corridors study}

As the discussion above indicates, the operations were designed such that equipped aircraft within corridors could largely be ignored if there was no crossing traffic, while unequipped aircraft still had to be communicated with. These differences had to be captured in the TBX computations. We used simple adjustment equations for both, but the equipped aircraft in the corridors adjustment was implemented as a primary multiplicative effect, whereas the adjustment for unequipped in corridors was implemented as a secondary additive adjustment

\section{Equipped Aircraft in Corridors:}

$$
p x_{\text {EinC }}(s, t)=1-\frac{a c_{\text {EinC }}(s, t)}{M A P}
$$

where

$p x_{\text {EinC }}$ is a primary complexity adjustment for the equipped aircraft in the corridors of sector $\mathrm{s}$ at time $\mathrm{t}$

$a c_{\text {EinC }}$ is the number of equipped aircraft predicted to be inside the corridors

\section{Unequipped Aircraft in Corridors:}

$$
s x_{\operatorname{UinC}}(s, t)=1-\frac{a c_{U i n C}(s, t)}{M A P}
$$

where

$p x_{\text {UinC }}$ is a secondary complexity adjustment for the unequipped aircraft in the corridors

$a c_{\text {UinC }}$ is the number of unequipped aircraft predicted to be inside the corridors

Please note that using the MAP value in the denominator can cause negative values if more aircraft enter the corridors than the MAP value. Therefore, the adjustment is not in line with the earlier requirement to stay within 0 and 2 . However, the study was designed such that the corridors traffic would never reach the MAP value, thereby keeping the adjustments to be positive. The effect of the corridors was likely overemphasized in any case and a more appropriate denominator than MAP might have been the actual aircraft count ac $(s, t)$.

\section{TBX formulation for corridors study}

Equation (11) shows the TBX formula used during the corridors study in its entirety.

The MAP value was set to 22 aircraft, and the equipage ratio to $50 \%$. Weather was not used in this study; therefore the weather adjustment equaled 1 and had no impact and so was not included in equation (11). In this study all weights in the secondary complexity adjustments were set to be 1 .

\section{Results}

The main results of the Corridors study will be presented in a future publication. We use only few data from the study here to show where TBX tracked well and where it needed to be improved.

\section{Metrics}

As metrics of interest for this discussion we use the controller workload, the aircraft count and the $T B X$ value. For this discussion we use the controller workload that was measured on the Radar position. The measurement was based upon the ATWIT technique [12]. Controllers were prompted every 3 minutes to rate their workload on a scale of 1 to 6 , given the guidelines indicated in Figure 4. Their rating was then recorded by the MACS data collection system. In the guidelines, we define the "in the Groove" rating as 3 and 4 and consider this to be the desirable level of workload. Ratings of 1 and 2 are too low and reflect inefficiencies in controller resource utilization. Ratings of 5 and 6 are too high as the controller may not be able to use their best judgment.

$$
\begin{aligned}
t b x(s, t)= & a c(s, t) * p x_{s v}(s, t) * p x_{E i n C}(s, t)+ \\
& a c_{n o m}(s) *\left(\frac{s x_{t r s}(s, t)+s x_{\text {PrLos }}(s, t)+s x_{\text {conf }}(s, t)+s x_{\text {uneq }}(s, t)+s x_{\text {UinC }}(s, t)}{5}-1\right)
\end{aligned}
$$




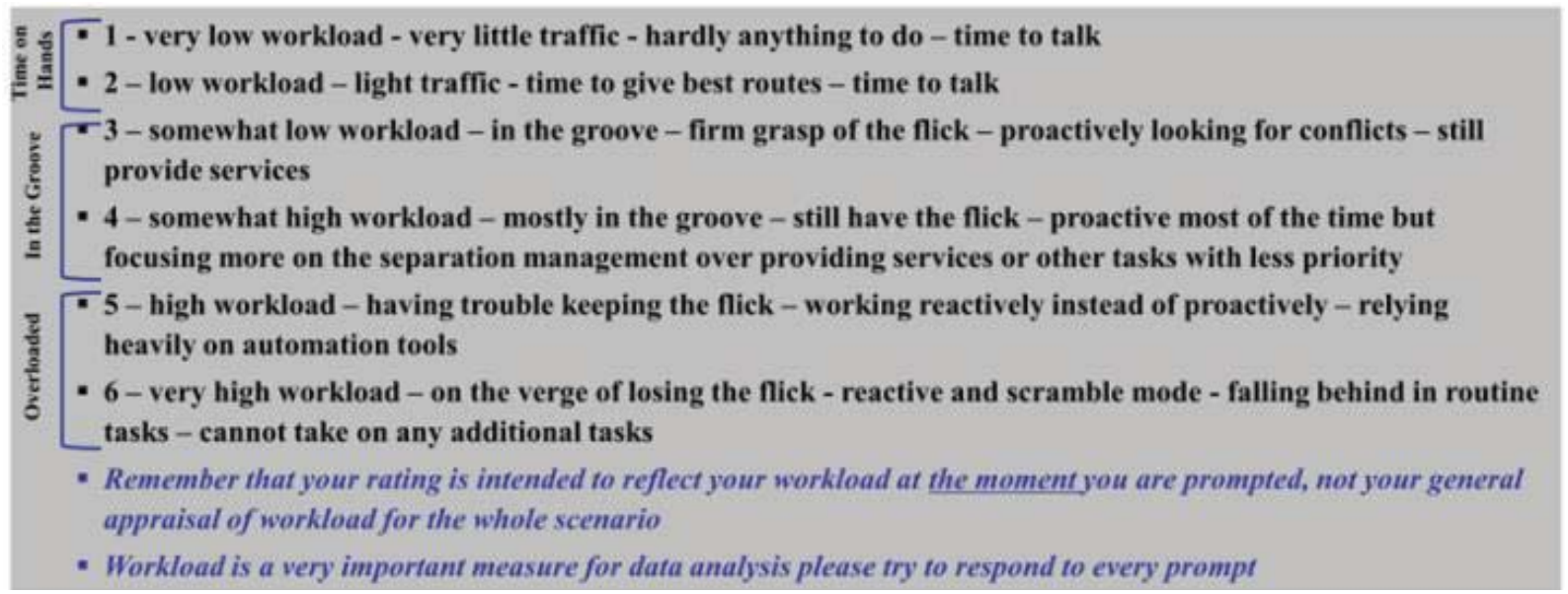

Figure 4: Guidelines given to the controllers for rating their workload on a scale of 1 to 6

We define aircraft count as the number of aircraft that are within the lateral and vertical boundaries of the sector of interest. This data was collected during the runs by the MACS built-in data collection system. Given that the goal is to keep the controller workload at 4 or less, the maximum allowable aircraft count under nominal conditions should correspond to a workload rating between 4 and 5. The MAP value was established at 22 aircraft for the nominal conditions that were defined with no corridors and a $50 \%$ equipage ratio.

The TBX value was computed in real-time as indicated in equation (11). In line with the prior discussion and the definition of TBX, a TBX value of 22 would reflect the maximum permissible workload under all conditions.

We will now look at the results of 2 sectors with very different characteristics (see Figure 2). Sector

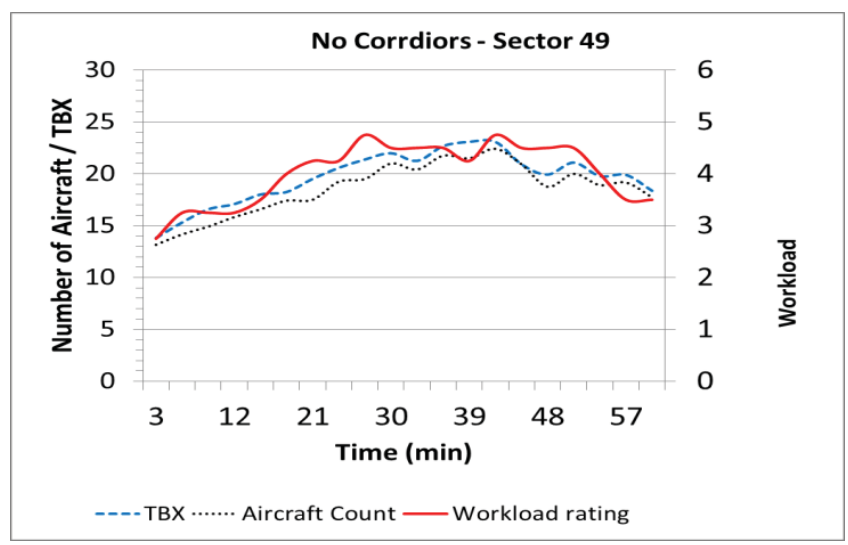

Figure 5: TBX, aircraft count and workload for sector 49 in $\mathrm{NC}$ condition
49, which is nearly square, has only one corridor in the corridors condition and feeds traffic into the corridors. Sector 79 accommodates two corridors and has a very different shape. We will first discuss the no corridors condition (NC) and then the condition of equipped aircraft in corridors (EinC).

\section{No Corridors (NC) condition}

The No corridors condition was considered as our nominal condition. Therefore, in this condition aircraft count was considered a good predictor of complexity and we expected the TBX value to be very close to aircraft count. Figure 5 shows workload rating, aircraft count and $\mathrm{TBX}$ rating for the $\mathrm{NC}$ condition in sector 49. As expected, both TBX and aircraft count were fairly good predictors for controller workload. Figure 6 shows similar results for sector 79 .

Both plots are laid out such that the MAP value

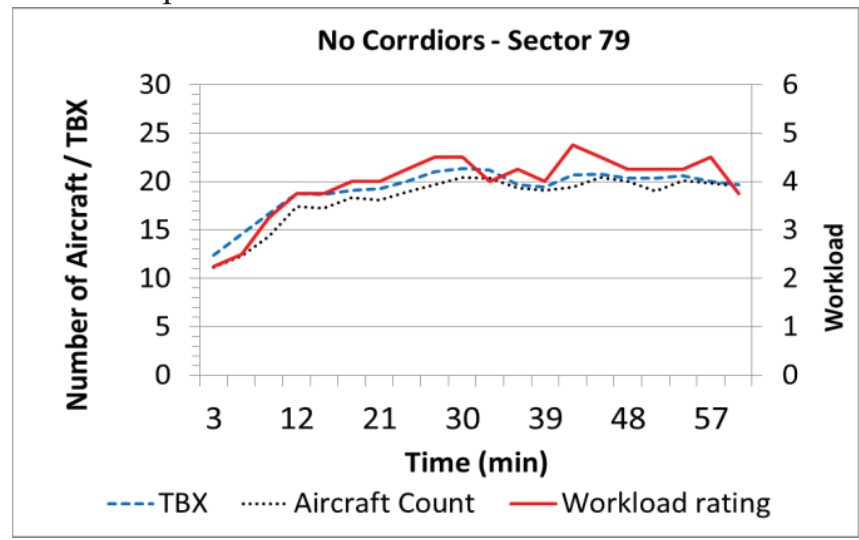

Figure 6: TBX, aircraft count and workload for sector 79 in NC condition 
of 22 crosses the workload axis between 4 and 5 . This means that as long as TBX is kept below MAP it is expected that the workload will be below 5. TBX, aircraft count and workload align well in both sectors. Both, TBX and aircraft count are usable predictors of controller workload with the TBX predictions being slightly higher and better than aircraft count. In the case of sector 49, TBX computed a value exceeding MAP about 40 minutes into the run that the aircraft count did not show. Otherwise, since the NC condition basically reflected our nominal case, TBX and aircraft count are very similar.

It should also be noted that the traffic managers and supervisors used TBX predictions in these runs to actively manage controller workload. The scenarios were designed to reach aircraft counts of up to 30 aircraft and the operators actively rerouted aircraft and changed their altitude to keep the workload manageable. Therefore, the result indicated a high but not excessive workload throughout the simulation run. This is not a coincidence but rather a consequence of actively managing traffic and resources based upon the TBX predictions.

\section{Equipped in Corridors (EinC)}

We use the "Equipped in Corridors" (EinC) condition as the second condition for our discussion because it is the condition that had the biggest differences from our nominal (NC) case. The complexity hypothesis for the EinC condition was that equipped aircraft in corridors could practically be ignored by the controllers due to an assumption that they required little monitoring effort and did not require clearances to issue for the controllers. This is reflected in our adjustment made for equipped aircraft in corridors. Equation (9) means that equipped aircraft in corridors can essentially be

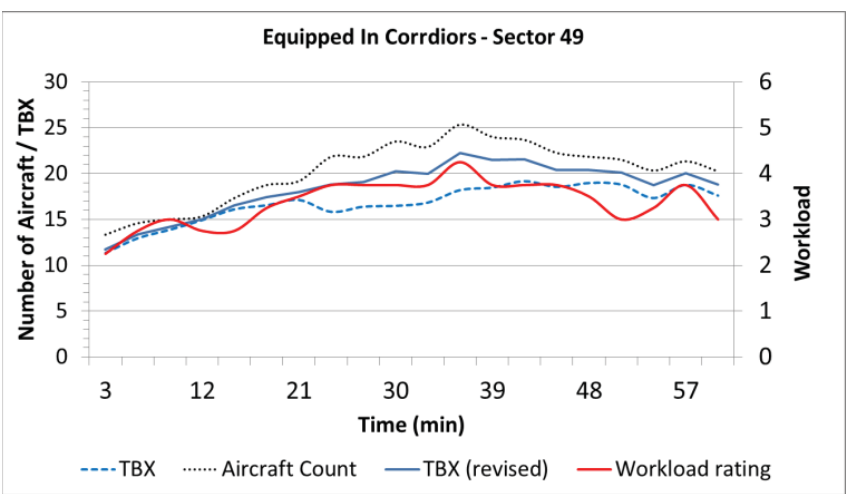

Figure 7: TBX, aircraft count, revised TBX and workload for sector 49 in EinC condition removed from the aircraft count to compute TBX when the overall aircraft count is around MAP.

The plots in Figures 7 and 8 show the aircraft count, TBX and workload results for the EinC condition. The aircraft count for both sectors reaches 25 and exceeds the MAP value for sustained periods of time. The workload however, hardly ever exceeds 4 is therefore well within the manageable range. Using only aircraft count, several aircraft would have been therefore unnecessarily removed from the sectors. While the TBX value for sector 49 appeared to be only slightly under-predicting the workload, it under-predicted the workload substantially for sector 79. The difference is due to the much larger amount of aircraft on corridors in sector 79 and the fact that a D-Side was added to sector 49 usually at about 35 minutes which immediately caused a workload reduction for the R-Side.

However, the data for sector 79 (Figure 8), show that the TBX value was clearly too low for this condition and did not accurately reflect the controllers' workload for the EinC condition. In interviews with controllers and during observations it was noted that the equipped aircraft in Corridors could only be ignored if there was no traffic trying to cross the corridors (usually on a climb trajectory) and if the corridors traffic did not have to exit the corridors within the sectors. Both of these conditions were only true for approximately half the aircraft on the corridors. Therefore, after the study we corrected our adjustment for Equipped in Corridors as follows:

$p x_{\text {EinC }}(s, t)=1-0.5 * \frac{a c_{E i n C}(s, t)}{M A P}$

where

$p x_{\text {EinC }}$ is a primary complexity adjustment for the equipped aircraft in the corridors of

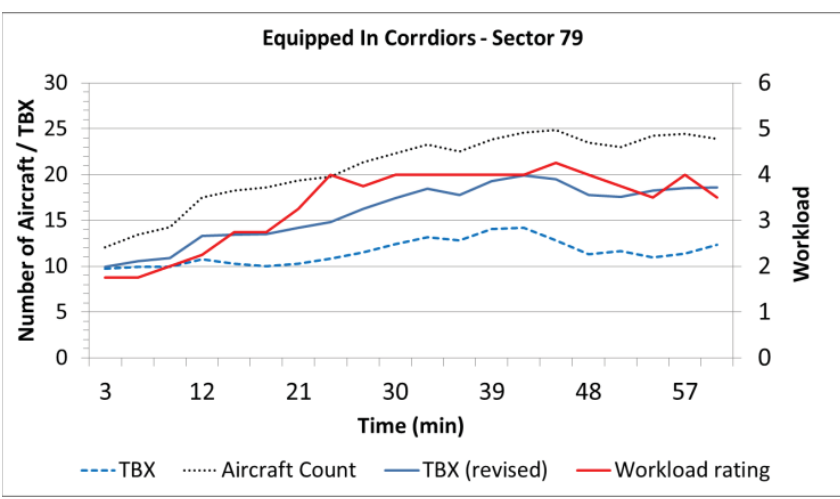

Figure 8: TBX, aircraft count, revised TBX and workload for sector $\mathbf{7 9}$ in EinC condition 
sector $\mathrm{s}$ at time $\mathrm{t}$

$a c_{\text {EinC }}$ is the number of equipped aircraft predicted to be inside the corridors

Using this adjustment we recomputed the TBX value and plotted it for both sectors as "TBX (revised)". The revised TBX matches the controller workload much better. To address the remaining mismatches we could adapt the secondary adjustments and weights of transitioning aircraft and conflicts to the respective sectors. We believe by doing so, we would get an extremely good TBX prediction for all sectors and condition. However, it will never be perfect and it does not have to be, as long as it can predict potentially difficult situations in time for the operators to make informed decisions.

\section{Conclusion}

We have developed a new complexity metric aggregate called TBX. TBX is a modified aircraft count for trajectory-based operations. TBX uses a simple framework to adjust the aircraft count according to variations of tractable and predictable primary and secondary complexity factors. We have used TBX during a number of human-in-the-loop simulations over the past few years, and TMCs and area supervisors were able to successfully manage controller resources and workload with TBX. TBX computations can easily be iterated and adjusted to the specific properties of sectors and operations and can improve the efficiency of traffic and resource management decisions.

\section{References}

[1] Joint Planning and Development Office, 2007, Concept of Operations for the Next Generation Air Transportation System, version 2.0.

[2] Prevot, T., P. Lee, T. Callantine, J. Mercer, J. Homola, N. Smith, \& E Palmer, 2010, Human-in-the-loop Evaluation of NextGen Concepts in the Airspace Operations Laboratory, AIAA Modeling and Simulation Technologies Conference and Exhibit, 2 - 5 August 2020, Toronto, Canada.

[3] Lee, P. U., N. Smith, T. Prevot, and J. R. Homola, 2010, Managing Demand and Capacity using Mulit-Sector Planning and Flexible Airspace: Human-In-The-Loop
Evaluation, 27th International Congress of the Aeronautical Sciences (ICAS), Nice, France.

[4] Kopardekar, P. and S. Magyarits, 2003, Measurement and Prediction of Dynamic Density, 5th USA/Europe Air Traffic Management $R \& D$ Seminar, Budapest, Hungary.

[5] Chatterji, G. B. and B. Sridhar, 2001, Measures for Air Traffic Controller Workload Prediction, 1st AIAA Aircraft Technology, Integration, and Operations (ATIO) Conference, Los Angeles, CA.

[6] Klein, A., M. D. Rogers, and K. Leiden, 2009, Simplified Dynamic Density: A Metric for Dynamic Airspace Configuration and NextGen Analysis, 28th Digital Avionics Systems Conference, Fairfax, VA.

[7] Masalonis, A. J., M.B. Callaham, and C.R. Wanke, 2003, Dynamic Density and Complexity Metrics for Realtime Traffic Flow Management, 5th USA/Europe Air Traffic Management $R \& D$ Seminar, Budapest, Hungary.

[8] Bloem, M., C. Brinton, J. Hinkey, K. Leiden, and K. Sheth, 2009, A Robust Approach for Predicting Dynamic Density, 9th AIAA Aircraft Technology, Integration, and Operations (ATIO) Conference, Hilton Head, SC.

[9] Hilburn, B. and G. Flynn, 2005, Modeling Air Traffic Complexity: Eurocontrol's COCA Approach, 24th Digital Avionics Systems Conference, Crystal City, Washington D. C.

[10] Histon, J. M. and R. J. Hansman, 2008, Mitigating Complexity in Air Traffic Control: The Role of Structure-based Abstractions, Technical Report, ICAT2008-05, Massachusetts Institute of Technology, Cambridge, MA.

[11] Li, L., 2009, Experimental Studies of Cognitively Based Air Traffic Control Complexity Metrics for Future Operational Concepts, Master's Thesis, Massachusetts Institute of Technology, Cambridge, MA. 
[12] Stein, E. S., 1985, Air traffic controller workload: An examination of workload probe, (DOTIFAA/ CT-TN84124). Atlantic City International Airport, NJ, Federal Aviation Administration Technical Center.

[13] Smith,N. M., C. L. Brasil, J. R. Homola, A. M. Kessell, H. E. Lee, P. U. Lee, M. J. Mainini, and J. S. Mercer,2011, A Humanin-the-Loop Evaluation of Flow-Based Trajectory Management in Mixed Equipage Airspace, Ninth USA/Europe Air Traffic Management Research and Development Seminar, ATM2011 (Paper 132), EUROCONTROL/FAA. Berlin, Germany, June 2011

\section{Acknowledgements}

We thank the team in the Airspace Operation Laboratory at NASA Ames for their many contributions to the research described in this paper. Special thanks go to the corridors and MSP research teams including Jeff Homola, Joey Mercer, Lynne Martin, Connie Brasil, Chris Cabrall, Hwasoo Lee, Matt Mainini and Nancy Smith. We appreciate the excellent work of the AOLs development team including Al Globus, Rick Jacoby, James Wong and Vick Kelkar.

Additional thanks go to NASA's Airspace Systems program and our FAA collaborators, as well as the many subject matter experts who provided their insights and participated in the simulations.

30th Digital Avionics Systems Conference

October 16-20, 201 